\title{
Chronic Copper Sulfate Poisoning
}

\author{
Ana Paula Perestrelo*, Gonçalo Miranda*, Maria Inês Gonçalves*, Carolina Belino, Rosa Ballesteros \\ Centro Hospitalar Universitário Cova da Beira, Alameda Pêro da Covilhã, Covilhã, Portugal \\ * Ana Paula Perestrelo, Gonçalo Miranda and Maria Inês Gonçalves contributed equally to this work as first authors.
}

Received: 19/01/2021

Accepted: $31 / 01 / 2021$

Published: $17 / 02 / 2021$

How to cite this article: Perestrelo AP, Miranda G, Gonçalves MI, Belino C, Ballesteros R. Chronic copper sulfate poisoning. EJCRIM 2021;8: doi:10.12890/2021_002309.

Conflicts of Interests: The Authors declare that there are no competing interests.

This article is licensed under a Commons Attribution Non-Commercial 4.0 License

\section{ABSTRACT}

Copper, as a salt, is toxic and has the potential to harm multiple organs. Copper intoxication causes intravascular haemolysis followed by liver and kidney failure that can be fatal. We present a case of chronic copper sulfate poisoning in a 66-year-old man with dysphagia to solids and liquids, anaemia, acute kidney injury, liver cytolysis and hypocalcaemia. The patient improved with supportive care, but chronic kidney disease was established. Anamnesis was crucial for the diagnosis, given the non-specific signs and symptoms. The history of chronic exposure to pest treatment with a blue dust cloud made us suspect copper sulfate poisoning.

\section{LEARNING POINTS}

- Copper sulfate poisoning is a relatively uncommon illness nowadays but can still be seen in clinical practice and should be kept in mind.

- It has non-specific signs and symptoms such as dysgeusia, abdominal pain, vomiting, muscle cramps or spasms, diarrhoea, ink-like urine, jaundice, anaemia and seizures.

- The diagnosis is based on clinical presentation and laboratory examinations upon a history of exposure to copper sulfate-containing products.

\section{KEYWORDS}

Copper sulfate poisoning, kidney injury

\section{CASE DESCRIPTION}

A 66-year-old man with history of type 2 diabetes mellitus, dyslipidaemia, benign prostatic hyperplasia and obstructive sleep apnoea was admitted to the emergency department for a 3-day history of dysphagia for solids and liquids. The patient also reported reduced urine output, dysgeusia, cramps, spasms and muscle weakness.

At admission, vital signs were normal but the oropharynx was difficult to observe due to trismus. Abdominal palpation showed diffuse tenderness without guarding. The patient also had muscle spasms in the lower limbs and fingers. The physical exam was otherwise unremarkable. The electrocardiogram was normal. Blood tests showed normochromic normocytic anaemia (haemoglobin $10.8 \mathrm{~g} / \mathrm{dl}$ ), acute kidney injury (urea $205 \mathrm{mg} / \mathrm{dl}$, creatinine $6.42 \mathrm{mg} / \mathrm{dl}$ ) and liver cytolysis (aspartate aminotransferase $162 \mathrm{U} / \mathrm{l}$, alanine aminotransferase 143 $\mathrm{U} / \mathrm{I}$ ). Arterial blood gas analysis showed hypocalcaemia (ionized calcium $2.57 \mathrm{mg} / \mathrm{dl}$ ). Because of the dysphagia, an upper digestive endoscopy was performed but showed no abnormalities. Calcium replacement with intravenous calcium gluconate was initiated and the patient was admitted to the internal medicine ward for further study and treatment.

Bladder and kidney ultrasound was performed. This excluded an obstructive cause for the kidney injury, and there were no signs of chronic kidney disease. After a detailed anamnesis, the patient reported that a neighbour carried out frequent pest treatment in his orchard with 
copper sulfate (described as a big blue dust cloud, with which the patient had close contact). Copper poisoning would explain the anaemia, hepatic dysfunction and kidney injury. He had 1.7\% methaemoglobin, serum copper $139 \mu \mathrm{g} / \mathrm{dl}$ (normal 70-140 $\mu \mathrm{g} / \mathrm{dl}$ ) and urinary copper $34 \mu \mathrm{g}$ in a $24 \mathrm{~h}$ urine collection (normal $15-50 \mu \mathrm{g}$ ). Although the copper values were normal to high, it was considered that the patient had already excreted most of the copper as this study was performed on the fourth day of his hospital stay. Hence, a diagnosis of chronic copper poisoning was assumed. The Poison Information Centre was contacted and it was decided not to initiate copper chelation therapy as the patient was improving with supportive care. Due to worsening anaemia, one blood unit was transfused with good response. Hypocalcaemia was corrected and muscle complaints regressed. The patient maintained good diuresis, renal function improved during his hospital stay and he was discharged.

During follow-up, chronic kidney disease was established and the anaemia resolved.

\section{DISCUSSION}

Copper is an essential trace element that is required for several important biological processes. Copper is not harmful in its metallic form, but as a salt it is toxic and has the potential to harm multiple organs. In its salt form it is used in fungicides, ceramics, fireworks, emetics and burn ointment. As a fungicide, combined with slaked lime, it is used in vineyards, farms and gardens to prevent infestations. It is considered a substance of particular concern to public health and the environment according to the European Food Safety Authority and its use is to be phased out and replaced ${ }^{[1]}$. Any manipulation of this compound without adequate protective gear can lead to poisoning. The acceptable daily intake of copper is $0.15 \mathrm{mg} / \mathrm{kg}$ of body weight per day ${ }^{[1]}$. Copper poisoning can result from inhalation, ingestion or topical contact with its salts. Clinically, it manifests with non-specific signs and symptoms, such as dysgeusia, abdominal pain, vomiting, muscle cramps or spasms, diarrhoea, ink-like urine, jaundice, anaemia and seizures ${ }^{[2,3]}$.

Copper intoxication causes intravascular haemolysis followed by liver and kidney failure that can be fatal. Haemolytic anemia occurs in the first 12-24 hours with oxidation of the heme group to form methaemoglobin. This process causes cyanosis and chocolate-colored blood ${ }^{[3,4]}$. Liver damage occurs on the second to third days. The patient may experience jaundice due to liver damage and haemolysis. Liver failure occurs due to mitochondrial dysfunction, necrosis and hepatocyte obstruction. Bilirubin elevation is directly proportional to severity. Liver biopsy typically shows centrilobular necrosis, mononuclear infiltrate and biliary stasis ${ }^{[3,4]}$. Acute kidney injury is a common manifestation that occurs on the third to fourth days. It is due to a combination of direct copper toxicity on proximal tubules, pre-renal failure caused by dehydration (vomiting, diarrhoea, reduced fluid intake) and intravascular haemolysis ${ }^{[4,5]}$.

Diagnosis is made by measuring serum ceruloplasmin and urinary copper, although it may be non-specific. It is important to assess methaemoglobin as it correlates with severity ${ }^{[6]}$. Renal failure must be recognized early by careful monitoring of serum creatinine and urine output. The recovery of renal function is slow and sometimes incomplete ${ }^{[3]}$.

Treatment in case of ingestion should avoid emetic measures as they can cause or aggravate oesophageal injuries. In some cases, activated carbon can be used. If the patient presents with methaemoglobinaemia, methylene blue may be used as well as hyperbaric oxygen therapy. Chelators, such as penicillamine and dimercaprol, are used only in an acute phase. Dialysis is ineffective in clearing copper but may be essential in acute kidney injury ${ }^{[2-4]}$.

Copper poisoning can lead to severe and life-threatening multi-organ dysfunction. In our case, anamnesis was crucial for the diagnosis, given the non-specific signs and symptoms. The history of chronic exposition to pest treatment with a blue dust cloud made us suspect copper sulfate poisoning. Despite the normal to high values of copper in blood, the diagnosis of copper poisoning was assumed as it explains the clinical condition and there is no linear relationship between copper levels and clinical expression ${ }^{[7]}$.

\section{REFERENCES}

1. Arena M, Auteri D, Barmaz S, Bellisai G, Brancato A, Brocca D, et al. Peer review of the pesticide risk assessment of the active substance copper compounds copper(I), copper(II) variants namely copper hydroxide, copper oxychloride, tribasic copper sulfate, copper(I) oxide, Bordeaux mixture. EFSA J 2018;16(1):e05152.

2. Gunay N, Yildirim C, Karcioglu O, Gunay NE, Yilmaz M, Usalan C, et al. A series of patients in the emergency department diagnosed with copper poisoning: recognition equals treatment. Tohoku J Exp Med 2006;209(3):243-248.

3. Gamakaranage CS, Rodrigo C, Weerasinghe S, Gnanathasan A, Puvanaraj V, Fernando H. Complications and management of acute copper sulphate poisoning; a case discussion J Occup Med Toxicol 2011;6(1):34.

4. Yang CC, Wu ML, Deng JF. Prolonged hemolysis and methemoglobinemia following organic copper fungicide ingestion. Vet Hum Toxicol 2004;46(6):321-323.

5. Faure A, Mathon L, Poupelin JC, Allaouchiche B, Chassard, D. Acute cupric sulfate intoxication: pathophysiology and therapy about a case report. Ann Fr Anesth Reanim 2003;22(6):557-559.

6. Franchitto N, Gandia-Mailly P, Georges B, Galinier A, Telmon N, Ducassé JL, et al. Acute copper sulphate poisoning: a case report and literature review. Resuscitation 2008;78(1):92-96.

7. Naha K, Saravu K, Shastry BA. Blue vitriol poisoning: a 10-year experience in a tertiary care hospital. Clin Toxicol 2012;50(3):197-201. 